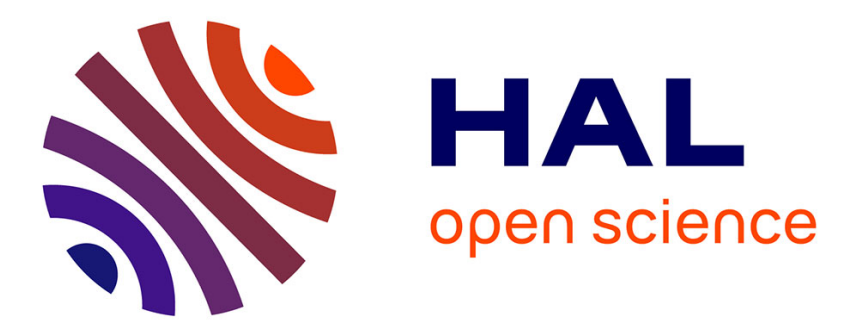

\title{
Temperature enhancement of the photorefractive effect in GaAs due to the metastable state of the EL2 defect
}

\author{
Philippe Delaye, B. Sugg
}

\section{To cite this version:}

Philippe Delaye, B. Sugg. Temperature enhancement of the photorefractive effect in GaAs due to the metastable state of the EL2 defect. Physical Review B: Condensed Matter and Materials Physics (1998-2015), 1994, 50 (23), pp.16973-16984. 10.1103/PhysRevB.50.16973 . hal-00673556

\section{HAL Id: hal-00673556 \\ https://hal-iogs.archives-ouvertes.fr/hal-00673556}

Submitted on 24 Feb 2012

HAL is a multi-disciplinary open access archive for the deposit and dissemination of scientific research documents, whether they are published or not. The documents may come from teaching and research institutions in France or abroad, or from public or private research centers.
L'archive ouverte pluridisciplinaire HAL, est destinée au dépôt et à la diffusion de documents scientifiques de niveau recherche, publiés ou non, émanant des établissements d'enseignement et de recherche français ou étrangers, des laboratoires publics ou privés. 


\title{
Temperature enhancement of the photorefractive effect in GaAs due to the metastable state of the $E L 2$ defect
}

\author{
Ph. Delaye \\ Institut d'Optique Théorique et Appliquée, Unité de Recherche Associée 14 au Centre National de la Recherche Scientifique, \\ Centre Scientifique d'Orsay, Bâtiment 503, Bôte Postale 147, 91403 Orsay Cedex, France \\ and Fachbereich Physik der Universität Osnabrück, 49069 Osnabrück, Germany \\ B. Sugg \\ Fachbereich Physik der Universität Osnabrück, 49069 Osnabrück, Germany \\ (Received 8 April 1994; revised manuscript received 12 September 1994)
}

\begin{abstract}
We present a theoretical and experimental analysis of photorefractive two-beam coupling in undoped GaAs as a function of temperature. The theoretical treatment includes the metastable state of the $E L 2$ defect with its optical properties (optical generation and optical recovery) in the photorefractive model. Three major features are predicted by this model: First, a change of the sign of the photorefractive beam coupling gain around $150 \mathrm{~K}$; second, an enhancement of the space-charge field by a factor of 2 compared to the diffusion field; and, finally, the appearance of a strong peak due to an absorption grating around $150 \mathrm{~K}$. All these features are actually observed experimentally with a good correlation between experimental data and theoretical simulation.
\end{abstract}

\section{INTRODUCTION}

The photorefractive effect (PRE) in a particular material depends on both its extrinsic defect-related and intrinsic properties: ${ }^{1}$ photoconductivity and carrier diffusion properties on one side, and the electro-optic coefficients, dielectric constants and the refractive index on the other. Photoconductivity is responsible for the creation of a space-charge field by a spatial redistribution of the trapped charges in the deep level due to a nonuniform illumination by photons of below-band-gap energy. The free carriers, created by photoionization of deep levels in the bright region of the illumination pattern, take part in the redistribution of the charges through diffusion (no external applied field) and/or drift (under external applied field) towards the dark region where they recombine. The redistribution of charges results in a spatially modulated space-charge field which is transformed in a spatially modulated refractive index variation via the electrooptic effect.

Important parameters which influence the PRE are the nature and characteristics of the deep defect in which carriers are redistributed. Its concentration and occupancy ratio as well as its optical cross sections greatly influence the strength of the PRE. Many studies are currently being performed in order to identify the deep level involved in the PRE in the different materials sensitive to the infrared [GaAs, ${ }^{2}$ InP, ${ }^{3,4}$ and CdTe (Ref. 5)]. Standard electrical methods of characterization of the deep levels such as deep-level transient spectroscopy (DLTS) are inapplicable in the high resistive samples used in PRE. To that purpose an optical contactless technique was proposed: deep-level photodiffractive spectroscopy (DLPS). ${ }^{6}$ In this technique the study of the variation of the strength of the PRE with temperature provides information about the deep levels present in the sample. This technique was used in InP:Fe to deduce the presence of a secondary defect that influences the PRE. ${ }^{4}$ In undoped GaAs, due to peculiar metastability properties of the EL 2 defect, drastic variations of the PRE at low temperature were expected and experimentally observed. $^{7,8}$

The aim of this paper is to present both theoretical and experimental investigations performed on GaAs:EL 2 with the DLPS technique. After a brief presentation of the $E L 2$ defect and its metastable state, in Sec. III we extend the photorefractive model in GaAs to low temperature, taking into account the optical and thermal properties of the metastable state of $E L 2$. The model predicts a peak of resonance of the photorefractive effect around $130 \mathrm{~K}$ with a created space-charge field higher than the diffusion field $E_{d}$. This peak follows a reverse of the sign of the PRE around $150 \mathrm{~K}$. All the theoretical features predicted were experimentally observed in two-beam coupling experiments at $1.047 \mu \mathrm{m}$ in the temperature range 90-300 K, as can be seen in Sec. IV.

\section{THE EL 2 DEFECT AND ITS METASTABLE STATE}

In liquid-encapsulated-Czochralski-(LEC)-grown undoped GaAs the semi-insulating character of the material is due to the compensation of the residual shallow donors and acceptors, with concentrations $N_{A}>N_{D} \approx 10^{15} \mathrm{~cm}^{-3}$, by the intrinsic $E L 2$ defect $\left(N_{E L 2} \approx 1 . .2 \times 10^{16} \mathrm{~cm}^{-3}\right){ }^{9}$ It gives rise to a deep level at $0.75 \mathrm{eV}$ below the bottom of the conduction band, where the Fermi level is pinned. The $E L 2$ defect can then exist in a neutral state $E L 2^{\circ}$ or a single ionized state $E L 2^{+} .{ }^{10}$ The $E L 2$ defect is an arsenic antisite-related double donor defect. The electrical compensation and, related to this, the atomic structure of 
the $E L 2$ defect have been the object of numerous studies, and recent reviews are available. ${ }^{11,12}$ An unusual property of this defect is the existence at low temperature of a metastable state called $E L 2^{*}$ which is optically and elec- trically inactive. ${ }^{13}$ The transfer of the defect from the neutral state $E L 2^{0}$ to the metastable state is optically induced by photons with an energy around $1.18 \mathrm{eV} .^{13}$ With this transfer, known as the photoquenching of EL2,

TABLE I. Parameters of the $E L 2$ defect used in the theoretical calculations (see text for details).

\begin{tabular}{ccc}
\hline Name & Value & Reference \\
\hline
\end{tabular}

$S_{n}$ electron photoionization cross section

$1 \times 10^{-16}$

at $1.06 \mu \mathrm{m}\left(\mathrm{cm}^{2}\right)$

$S_{p}$ hole photoionization cross section at

$3 \times 10^{-17}$

$1.06 \mu \mathrm{m}\left(\mathrm{cm}^{2}\right)$

$\sigma_{n}$ capture cross section of electrons

$5 \times 10^{-19}+6 \times 10^{-15} \exp \left(-0.0566 \frac{e}{k_{B} T}\right)$

$\left(\mathrm{cm}^{2}\right)$

$\sigma_{p}$ capture cross section of holes $\left(\mathrm{cm}^{2}\right)$

$2 \times 10^{-18}$

$v_{n \text {th }}$ thermal velocity of electrons $\left(\mathrm{cm} \mathrm{s}^{-1}\right)$

$4.4 \times 10^{7}\left(\frac{T}{300}\right)^{1 / 2}$

$v_{p t h}$ thermal velocity of holes $\left(\mathrm{cm} \mathrm{s}^{-1}\right)$

$1.65 \times 10^{7}\left(\frac{T}{300}\right)^{1 / 2}$

$\mu_{n}$ electron mobility $\left(\mathrm{cm}^{2} \mathrm{~V}^{-1} \mathrm{~s}^{-1}\right)$

$$
8000\left(\frac{T}{300}\right)^{2.3}
$$

$\mu_{\rho}$ hole mobility $\left(\mathrm{cm}^{2} \mathrm{~V}^{-1} \mathrm{~s}^{-1}\right)$

$$
320\left(\frac{T}{300}\right)^{2.3}
$$

$\beta_{n}$ electron thermal emission coefficient

$$
2.83 \times 10^{7} T^{2} \exp \left(-0.814 \frac{e}{k_{B} T}\right)
$$

$\left(\mathrm{s}^{-1}\right)$

$\beta_{p}$ hole thermal emission coefficient $\left(\mathrm{s}^{-1}\right)$

$$
\begin{gathered}
3.3 \times 10^{4} T^{2} \exp \left[-0.813 \frac{e}{k_{B} T}\right) \\
2 \times 10^{11} \exp \left(-0.30 \frac{e}{k_{B} T}\right)
\end{gathered}
$$

$\left(s^{-1}\right)$

$S_{n}^{*}$ metastable state generation optical $1 \times 10^{-17}$ cross section $\left(\mathrm{cm}^{2}\right)$

$S_{r}^{*}$ recovery optical cross section of the metastable state $\left(\mathrm{cm}^{2}\right)$

$\varepsilon_{r}$ relative dielectric constant

$$
12.4\left(1+1.2 \times 10^{-4} \mathrm{~T}\right)
$$

$n_{0}$ refractive index

$$
\begin{gathered}
n_{0}^{2}=A+\left[\frac{n_{\infty}^{2}-A}{1-B(h v)^{2}}\right] \\
A=7.10 \\
B=0.18(\mathrm{eV})^{-2} \\
n_{\infty}=3.255\left(1+4.5 \times 10^{-5} T\right) \\
h v \text { in } \mathrm{eV}
\end{gathered}
$$


many of the characteristics of $E L 2$ disappear such as below-band-gap absorption, ${ }^{14}$ electron paramagnetic resonance spectrum, ${ }^{15}$ and diffraction efficiency in PRE measurements. ${ }^{7}$ Until now no model has satisfactorily explained this quenching of the DLPS signal. The aim of this work is to introduce the $E L 2^{*}$ metastable state in a band transport model ${ }^{16,17}$ that describes the PRE, and to observe the influence of this state on the DLPS signal.

All the properties of the defect used in the band transport model are summarized in the energy diagram of the EL2 defect (Fig. 1), and the values of the parameters (with their possible temperature dependence) are summed up in Table $I$. Carriers are generated from the $E L 2^{0} / E L 2^{+}$defect both thermally and optically with an electron thermal emission rate $\beta_{n}\left(\beta_{p}\right.$ for holes) and an electron photoionization cross section $S_{n}$ ( $S_{p}$ for holes), respectively. At the photon energy used in our experiments $(h v=1.18 \mathrm{eV})$, we are far from the photoionization threshold $(0.7-0.8 \mathrm{eV})$, thus the temperature dependence of the photoionization cross section is expected to be low and is neglected in the end of the study. This fact is confirmed by the small temperature variation observed in the absorption spectra in this wavelength region ${ }^{25}$ (at low illumination, without quenching). The carrier recombination is characterized by a recombination coefficient $\gamma_{n(p)}$ for electrons (for holes). These parameters are written $\gamma_{n(p)}=\sigma_{n(p)} v_{n(p) \text { th }}$ as a function of the thermal velocity $v_{n(p) \text { th }}$ and the capture cross sections $\sigma_{n(p)}$ of the carriers. All these parameters can be temperature dependent. The mobilities of electrons and holes are $\mu_{n}$ and $\mu_{p}$, respectively. The transfer from $E L 2^{0}$ to $E L 2^{*}$ is optically induced with a cross section $S_{n}^{*} .{ }^{13}$ The spectrum of $S_{n}^{*}$ is a sharp band centered at $1.18 \mathrm{eV}$ with a width of about $200-300 \mathrm{meV}$. The absolute value of the cross section at the maximum (our experiments are performed at this maximum) is about a tenth of the electron photoionization cross section $S_{n}$. As there is no evidence of any temperature variation, we assume $S_{n}^{*}$ to be constant. The recovery is induced both thermally with a recovery rate $r^{*}, 13$ and optically with an optical recovery cross section $S_{r}^{*} .^{22}$ Though the thermal recovery is well known (as we are in semi-insulating materials we neglect the "Augerlike" process in the presence of free electrons ${ }^{13}$ ), this is not the case of the optical recovery especially concerning

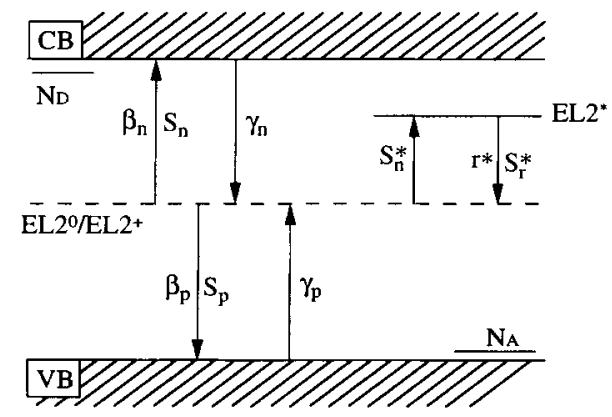

FIG. 1. Energy diagram of the $E L 2$ defect in GaAs as a function of a space coordinate. the wavelength around $1.18 \mathrm{eV}$. A lot of papers provide evidence of the existence of this optical recovery (for a review see Ref. 12). The spectrum of $S_{r}^{*}$ is characterized by two bands centered at 0.85 and $1.45 \mathrm{eV}$. Between these two maxima the cross section seems to go to a minimum for our energy of interest $(1.18 \mathrm{eV}) .^{22}$ No absolute value of this cross section is accessible, nevertheless we can estimate the ratio $S_{n}^{*} / S_{r}^{*}$ from the experimental curves of Ref. 22. The recovery of the quenched absorption measured at $1.18 \mathrm{eV}$ is about $1 \%$. This absorption recovery can be written as a function of the different cross sections as $S_{r}^{*} /\left(S_{r}^{*}+S_{n}^{*}\right),{ }^{26}$ which gives an estimation for the ratio $S_{n}^{*} / S_{r}^{*} \approx 100$. In this estimation it is supposed that the absorption recovery is entirely a competition between quenching and recovery. If other phenomena add to this competition (as suggested by Ref. 22) the ratio would be increased, but in the following this will not be considered. The optical recovery cross section was also found to be thermally activated. ${ }^{27}$ In our study the temperature range where the metastable state is studied is between 90 and $150 \mathrm{~K}$, a range where $S_{r}^{*}$ varies only slightly with temperature $^{27}$ (a factor 2-3), so we neglect this temperature effect to simplify the present study. The temperature variation is known ${ }^{27}$ and can be taken into account in further numerical simulations or for experiments at lower temperature.

Thus we see that all parameters that characterize the $E L 2$ defect and its metastable state are well known from the literature, including their temperature dependence. We will use these parameters to predict the behavior of the photorefractive effect in undoped GaAs at low temperature.

\section{THEORETICAL MODEL}

The total $E L 2$ concentration is $[E L 2]=N_{T}$, with an ionized part $E L 2^{+}$in concentration $\left[E L 2^{+}\right]=N$ and a neutral part $E L 2^{\circ}$ in concentration $\left[E L 2^{0}\right]$ $=N_{T}-N-N^{*},\left[E L 2^{*}\right]=N^{*}$ being the concentration of the defect in the metastable state $E L 2^{*}$. The electron and hole current densities are $\mathbf{j}_{n}$ and $\mathrm{j}_{p}$, and the free hole and electron densities are $p$ and $n$. $\mathbf{E}$ is the electric field inside the crystal. The material equations that are used to describe the band transport model ${ }^{16,17}$ of the PRE are the following.

The rate equations for $E L 2^{+}$and $E L 2^{*}$ concentrations are

$$
\begin{aligned}
\frac{\partial N}{\partial t}= & \left(\beta_{n}+S_{n} I\right)\left(N_{T}-N-N^{*}\right)-\gamma_{n} n N-\left(\beta_{p}+S_{p} I\right) N \\
& +\gamma_{p} p\left(N_{T}-N-N^{*}\right) \\
\frac{\partial N^{*}}{\partial t}= & S_{n}^{*} I\left(N_{T}-N-N^{*}\right)-\left(r^{*}+S_{r}^{*} I\right) N^{*}
\end{aligned}
$$

The current density equations are

$$
\begin{aligned}
& \mathrm{j}_{n}=e n \mu_{n} \mathrm{E}+\mu_{n} k_{B} T \nabla n, \\
& \mathrm{j}_{p}=e p \mu_{p} \mathbf{E}-\mu_{p} k_{B} T \nabla p .
\end{aligned}
$$

The continuity equations are 


$$
\begin{aligned}
& \frac{\partial n}{\partial t}=\frac{\nabla \cdot \mathrm{j}_{n}}{e}+\left(\beta_{n}+S_{n} I\right)\left(N_{T}-N-N^{*}\right)-\gamma_{n} n N \\
& \frac{\partial p}{\partial t}=\frac{-\nabla \cdot \mathrm{j}_{p}}{e}+\left(\beta_{p}+S_{p} I\right) N-\gamma_{p} p\left(N_{T}-N-N^{*}\right)
\end{aligned}
$$

The Poisson equation is

$$
\nabla \cdot \mathbf{E}=\frac{-e}{\varepsilon}\left(-N+N_{A}-N_{D}+n-p\right) .
$$

$I$ is a spatially modulated illumination $I(z)$ $=I_{0}[1+\operatorname{Re}(m \exp (i k z))]$ with $I_{0}$ as photon current in $\mathrm{s}^{-1} \mathrm{~cm}^{-2}$, a modulation $m$ of the fringes and the wave number $k$ of the fringes $(k=2 \pi / \Lambda, \Lambda$ the grating spacing). $e, \mu_{n}$, and $\mu_{p}$ are the absolute values of electron and hole charges and mobilities; $\varepsilon$ is the static dielectric constant of the material $\left(\varepsilon=\varepsilon_{0} \varepsilon_{r}\right)$; and $N_{A}$ and $N_{D}$ are the densities of compensative acceptors and donors, respectively.

Equations (3.1a)-(3.1g) form a set of equations which allow us to determine $N, N^{*}, n, p, j_{n}, j_{p}$, and $E$. Each one of them has a solution of the general form $X(z)=\left[X_{0}+\operatorname{Re}\left(\Sigma_{n>0} X_{n} \exp (\right.\right.$ inkz $\left.\left.)\right)\right]$. Considering the small modulation of the illumination pattern $(m<1)$, the usual linearization is performed, keeping only the zero- and first-order terms. In the steady state $(\partial X / \partial t=0)$ an analytical solution can be found. Similar equations have been solved by many authors and discussed under different aspects. ${ }^{28}$ We will present the solution for the space-charge field $E_{1}$, which is the important parameter for the PRE, under consideration of the metastable state $E L 2^{*}$, and will discuss it as a function of temperature.

\section{A. Zeroth-order solution}

First, we solve the equation for the nonmodulated term $X_{0}$, neglecting the influence of higher-order terms (of the order of $m X_{1}$ ) as a small modulation of the illumination pattern $^{16}$ is used. We obtain

$$
\begin{aligned}
& N_{T}-N_{0}-N_{0}^{*}=\left(N_{T}-N_{0}\right) A^{\prime *}, \\
& N_{0}^{*}=\left(1-A^{\prime *}\right)\left(N_{T}-N_{0}\right), \\
& n_{0}=\frac{\left(\beta_{n}+S_{n} I_{0}\right)\left(N_{T}-N_{0}\right) A^{\prime *}}{\gamma_{n} N_{0}}, \\
& p_{0}=\frac{\left(\beta_{p}+S_{p} I_{0}\right) N_{0}}{\gamma_{p}\left(N_{T}-N_{0}\right) A^{\prime *}}, \\
& N_{0}=N_{A}-N_{D}+n_{0}-p_{0},
\end{aligned}
$$

with

$$
A^{\prime *}=\frac{r^{*}+S_{r}^{*} I_{0}}{r^{*}+S_{r}^{*} I_{0}+S_{n}^{*} I_{0}}
$$

Replacing $n_{0}$ and $p_{0}$ by their expression as a function of $N_{0}$ in Eq. (3.6), we obtain a third-order equation that gives $N_{0}$. We then deduce all the other parameters using Eqs. (3.2)-(3.5). We see that for a fixed illumination $I_{0}$ all populations of the different species are perfectly determined.

We can simplify the problem in the case of semiinsulating materials. Indeed in that type of materials we know that, at room temperature, the free-electron and hole populations $n_{0}$ and $p_{0}$ are very low compared to defect concentrations, which means that Eq. (3.6) becomes $N_{0}=N_{A}-N_{D}$ (the compensation of the excess of shallow acceptors is assured by holes trapped in the $E L 2$ defect). With decreasing temperature the $E L 2$ defect is quenched, which results in a generation of free holes (and the material becomes $p$ type). Considering these two points we can reasonably consider that we can neglect the freeelectron concentration for the entire temperature range in semi-insulating samples. Then Eq. (3.6) becomes

$$
N_{0}=N_{A}-N_{D}-p_{0},
$$

and the resulting equation becomes a second-order equation in $N_{0}$ :

$$
\begin{array}{r}
N_{0}^{2}-N_{0}\left[\left[\frac{\beta_{p}+S_{p} I_{0}}{\gamma_{p} A^{\prime *}}\right]+\left(N_{A}-N_{D}+N_{T}\right)\right] \\
+\left(N_{A}-N_{D}\right) N_{T}=0 .
\end{array}
$$

The temperature dependence of this equation occurs through the first term of the second member of the equation and particularly $A^{\prime *}$. Indeed $A^{\prime *}$ depends on the recovery rate $r^{*}$ (Table I). At high temperature $r^{*}$ goes to a value much greater than $S_{r}^{*} I_{0}+S_{n}^{*} I_{0}$ and $A^{\prime *}$ equals approximately 1 . When temperature decreases, $r^{*}$ goes to zero and $A^{\prime *}$ decreases to a limit $A^{\prime *}=S_{r}^{*} /\left(S_{r}^{*}+S_{n}^{*}\right)$, which goes to zero as $S_{r}^{*}$ decreases with temperature. ${ }^{27}$ At room temperature, we have $\left[\left(\beta_{p}+S_{p} I_{0}\right) / \gamma_{p} A^{\prime *}\right]$ $\ll\left(N_{A}-N_{D}+N_{T}\right)$, and Eq. (3.8) gives $N_{0}=N_{A}-N_{D}$, as previously seen (or $N_{0}=N_{T}$, but this is physically meaningless). When the temperature decreases, $A^{\prime *}$ goes nearly to zero and the approximation $\left[\left(\beta_{p}+S_{p} I_{0}\right) / \gamma_{p} A^{\prime *}\right] \ll\left(N_{A}-N_{D}+N_{T}\right)$ is no longer valid, and $N_{0}$ decreases (Fig. 2). We can note that in the

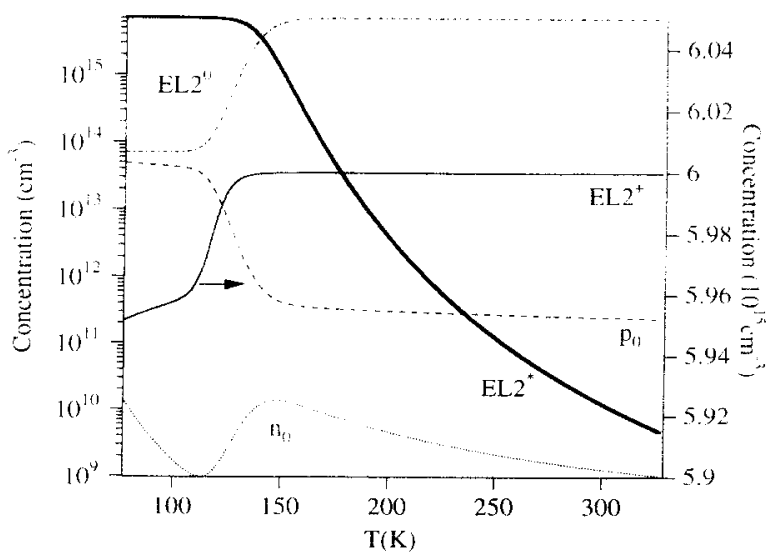

FIG. 2. Calculated concentration of the different species present in undoped $\mathrm{GaAs}$ as a function of the temperature (illumination $I=60 \mathrm{~mW} \mathrm{~cm}^{-2}$ ). 
temperature range $77 \mathrm{~K}<T<300 \mathrm{~K}$ the condition is verified and $N_{0}$ is almost constant with a value which equals $N_{A}-N_{D}$ (the room-temperature value) (Fig. 2). We also see in Fig. 2 that the free-electron concentration stays very low compared to $N_{0}$ for the entire temperature range, as supposed previously. At lower temperature $(T<77 \mathrm{~K})$, due to the temperature decrease of $S_{r}^{*}, N_{0}$ is quenched.

Here we have an important feature of the $E L 2$ quenching in GaAs, although $A^{\prime *}$ has decreased by a factor 100 , when passing from 300 to $77 \mathrm{~K}$ [which means that the $E L 2^{\circ}$ concentration has decreased by the same factor $\mathrm{Eq}$. (3.2)] the $E L 2^{+}$concentration has not changed. We have quenched only the neutral part of $E L 2$ and not the ionized part. This is easy to understand physically, as the transfer from $E L 2^{+}$to the metastable state is a transfer in two steps: first, the generation of a hole is accompanied by the transformation of $E L 2^{+}$to $E L 2^{0}$, then $E L 2^{\circ}$ is transferred to $E L 2^{*}$. Then there is competition between the lifetime of holes and the lifetime of the metastable state, resulting in a reduced efficiency of the transfer from $E L 2^{+}$to $E L 2^{*}$. We can also see in Fig. 2 that the concentration of free electrons remains negligible, and that free holes are generated at low temperature by the quenching of $E L 2^{+}$.

From Eq. (3.8) we deduce

$\left[E L 2^{+}\right]=N_{0}=\frac{1}{2}\left[\left(N_{A}-N_{D}+N_{T}\right)+\left(\frac{\beta_{p}+S_{p} I_{0}}{\gamma_{p} A^{\prime *}}\right)-\left\{\left[\left(N_{A}-N_{D}+N_{T}\right)+\left[\frac{\beta_{p}+S_{p} I_{0}}{\gamma_{p} A^{\prime *}}\right]\right]^{2}-4\left(N_{A}-N_{D}\right) N_{T}\right\}^{1 / 2}\right]$,

which immediately allows us to determine $\left[E L 2^{\circ}\right]$ using Eq. (3.2). The second solution of the equation is greater than $N_{T}$.

This feature of quenching will be very important for the strength of the PRE, as we will see now.

\section{B. First-order solution}

The equation for the spatially modulated terms $X_{1}$ is solved, neglecting, as usual, the influence of the freecarrier grating $\left(n_{1}\right.$ and $\left.p_{1}\right)$ (Ref. 17) in comparison with the deep trap grating $N_{1}$. In addition, we assume an externally applied field to be absent $\left(E_{0}=0\right)$. For the spatially modulated space-charge field $E_{1}$ (the calculation is given in the Appendix) we obtain

$E_{1}=\frac{-i m\left[\frac{k_{B} T}{e}\right) k}{1+A^{\prime *} \frac{k^{2}}{k_{0}^{2}}}\left[\xi(k)-\left(1-A^{\prime *}-B^{\prime *}\right)\right]$,

with $\xi(k)$ the electron-hole competition coefficient: ${ }^{17}$

$$
\xi(k)=\frac{\alpha_{n}\left(k^{2}+\kappa_{p}^{2}\right)-\alpha_{p}\left(k^{2}+\kappa_{n}^{2}\right)}{A_{n} \alpha_{n}\left(k^{2}+\kappa_{p}^{2}\right)+A_{p} \alpha_{p}\left(k^{2}+\kappa_{n}^{2}\right)} .
$$

$A^{\prime *}=\left(r *+S_{r}^{*} I_{0}\right) /\left(r^{*}+S_{r}^{*} I_{0}+S_{n}^{*} I_{0}\right)$ was previously defined, and $B^{\prime *}=S_{n}^{*} S_{r}^{*} I_{0}^{2} A^{\prime *} /\left[\left(r^{*}+S_{r}^{*} I_{0}\right)^{2}\right] . \quad A_{n}$ $=\left(\beta_{n}+S_{n} I_{0}\right) / S_{n} I_{0}$ and $A_{p}=\left(\beta_{p}+S_{p} I_{0}\right) / S_{p} I_{0}$ give the influence of the thermal emission of carriers from $E L 2$ at high temperature. $\alpha_{n}=S_{n}\left(N_{T}-N_{0}-N_{0}^{*}\right)$ and $\alpha_{p}=S_{p} N_{0}$ are the parts of the absorption that create electrons and holes, respectively. $\kappa_{n}^{2}=\left(e / k_{B} T\right)\left(\gamma_{n} N_{0} / \mu_{n}\right)$ and $\kappa_{p}^{2}=\left(e / k_{B} T\right)\left[\gamma_{p}\left(N_{T}-N_{0}-N_{0}^{*}\right)\right] / \mu_{p}$ are the inverse squared of the diffusion lengths of electrons and holes. Finally, $k_{0}^{2}=\left(e^{2} / \varepsilon k_{B} T\right)\left[N_{0}\left(N_{T}-N_{0}-N_{0}^{*}\right)\right] / N_{T}$ is the inverse squared of the Debye screening length. The different concentrations $N_{0}$ and $\left(N_{T}-N_{0}-N_{0}^{*}\right)$ are given by the zeroth-order solution of the equations, i.e., (3.9) and (3.2).

The space-charge field is purely imaginary, which corresponds to a grating that is $\pi / 2$ shifted with respect to the illumination grating.

At room temperature we have $A^{\prime *}=1$ and $B^{\prime *}=0$ then $\left(1-A^{\prime *}-B^{\prime *}\right)=0$ (as $r^{*} \gg S_{n}^{*} I_{0}, S_{r}^{*} I_{0}$ ) and we find the well-known expression of the space-charge field given by the electron-hole competition model. ${ }^{17}$

Considering the values of the different parameters at room temperature, we can make the usual approximation $\kappa_{n}^{2}, \kappa_{p}^{2} \ll k^{2}$. Moreover, as thermal emission is negligible for temperatures below $300 \mathrm{~K}$ we have $A_{n}=A_{p}=1$, and $\xi(k)$ reduces to

$$
\xi(k)=\xi_{0}=\frac{\alpha_{n}-\alpha_{p}}{\alpha_{n}+\alpha_{p}}=\frac{S_{n}\left[E L 2^{0}\right]-S_{p}\left[E L 2^{+}\right]}{S_{n}\left[E L 2^{0}\right]+S_{p}\left[E L 2^{+}\right]} .
$$

At low temperature $\kappa_{n}^{2}$ and $\kappa_{p}^{2}$ decrease, and the approximation is also justified, and we take the expression $\xi(k)=\xi_{0}$ in the following study (we will take the whole expression for the numerical simulations).

The influence of the metastable state of $E L 2$ appears in the $k$ dependence of $E_{1}$ with the term $k_{0}^{2} / A^{\prime *}$. Taking into account Eq. (3.2), we obtain

$$
\frac{k_{0}^{2}}{A^{\prime *}}=\frac{e^{2}}{\varepsilon k_{B} T} \frac{N_{0}\left(N_{T}-N_{0}\right)}{N_{T}}
$$

As $N_{0}\left(=\left[E L 2^{+}\right]\right)$stays constant between 77 and $300 \mathrm{~K}$, we see that the term $\left(k_{0}^{2} / A^{\prime *}\right)\left(k_{B} T / e\right)$ retains the value it has at $300 \mathrm{~K}$. Then, in this temperature range, the metastable state does not bring additional temperature variation to the $e / k_{B} T$ dependence of $k_{0}^{2}$ we can observe in the classical model. ${ }^{17}$ At lower temperature, $N_{0}$ decreases (as $S_{r}^{*}$ decreases) and $k_{0}^{2} / A^{\prime *}$ decreases and goes to zero, decreasing $E_{1}$. Then the only remaining 
influence of the metastable state between 77 and $300 \mathrm{~K}$ is through the term of "hole-electron" competition, i.e., through the value of $R=\xi_{0}-\left(1-A^{\prime *}-B^{\prime *}\right)$. We will now discuss the variation of this parameter with temperature, by considering its two terms independently to clarify the situation.

The electron-hole competition factor $\xi_{0}$ depends on both photoionization cross sections $S_{n}$ and $S_{p}$ and zeroth Fourier components of the concentrations of $E L 2^{\circ}$ and $E L 2^{+}$, namely $\left(N_{T}-N_{0}-N_{0}^{*}\right)$ and $N_{0}$. We saw that these concentrations varied with temperature due to the quenching of $E L 2$. The $E L 2^{\circ}$ density decreases by more than two orders of magnitude when the temperature goes below $150 \mathrm{~K}$, whereas the $E L 2^{+}$concentration stays constant until $77 \mathrm{~K}$ (Fig. 2). This means that the electronhole competition changes with temperature. In real cases, many different characteristics can be observed depending on the initial compensation ratio of the sample. In samples with a great excess of acceptors, i.e., $\left[E L 2^{0}\right] /\left[E L 2^{+}\right] \approx 1$, we observe electron-hole competition at room temperature at $1 \mu \mathrm{m}, \xi_{0}<1$ [with a reverse of the sign of the majority carrier at $1.32 \mu \mathrm{m}$ (Ref. 25)]. This is due to the higher value of $S_{n}$ compared to $S_{p}$ (Table I). When decreasing temperature $\left[E L 2^{0}\right]$ decreases and $\left[E L 2^{+}\right]$stays constant, the ratio becomes $\left[E L 2^{0}\right] /\left[E L 2^{+}\right] \approx 1 / 100$, and we observe a change of the sign of the electron-hole competition, the holes becoming majority carriers for the PRE. The change of the ratio is enough to completely reverse the competition and $\xi_{0}$ goes to -1 , and electrons have disappeared from the PRE $\left(S_{n}\left[E L 2^{0}\right] \ll S_{p}\left[E L 2^{+}\right]\right)$. The other extreme of GaAs samples is the class of crystals with a low excess of acceptors, and where we have $\left[E L 2^{0}\right] /\left[E L 2^{+}\right] \approx 10$. At room temperature in that kind of sample we have no electron-hole competition at $1 \mu \mathrm{m}$, and electrons are the majority carriers, $\xi_{0} \approx 1$ [no reverse of the sign of the majority carrier at $1.32 \mu \mathrm{m}$ (Ref. 25)]. With decreasing temperature the ratio inverts $\left[E L 2^{0}\right] /\left[E L 2^{+}\right] \approx 1 / 10$, and the electron hole competition increases, but not enough to have a complete change of the sign of the majority carriers. We obtain an almost exact compensation of the electrons by the holes, $\xi_{0} \approx 0\left(S_{n}\left[E L 2^{0}\right] \approx S_{p}\left[E L 2^{+}\right]\right)$. Between these two extremes we observe all the possible behavior with a general trend of change of the sign of the electron-hole competition coefficient $\xi_{0}$, which becomes negative.

We will now discuss the term $\left(1-A^{\prime *}-B^{\prime *}\right)$ which can be written as follows:

$$
\left(1-A^{\prime *}-B^{\prime *}\right)=\frac{S_{n}^{*} I_{0} r^{*}}{\left(r^{*}+S_{r}^{*} I_{0}+S_{n}^{*} I_{0}\right)\left(r^{*}+S_{r}^{*} I_{0}\right)} .
$$

At room temperature $r^{*} \gg S_{n}^{*} I_{0}, S_{r}^{*} I_{0}$; then $\left(1-A^{\prime *}-B^{\prime *}\right) \approx 0$. At low temperature $r^{*}$ goes to zero, and so does $\left(1-A^{\prime *}-B^{\prime *}\right)$, which means that $\left(1-A^{\prime *}-B^{\prime *}\right)$ goes through a maximum when we decrease temperature. If we suppose that the only temperature-varying parameter is $r^{*}$ (we suppose that $T>77 \mathrm{~K}$ and that $S_{r}^{*}$ is constant), the maximum is obtained for $r_{M}^{*}=I_{0} \sqrt{S_{r}^{*}\left(S_{r}^{*}+S_{n}^{*}\right)}$, the maximum value being

$$
\left(1-A^{\prime *}-B^{\prime *}\right)_{M}=\frac{S_{n}^{*}}{\left(\sqrt{S_{r}^{*}}+\sqrt{S_{r}^{*}+S_{n}^{*}}\right)^{2}} .
$$

From the value of $r_{M}^{*}$ (Table I) we deduce the temperature at which the maximum occurs. First, we remark that $r_{M}^{*}$ depends on the incident illumination. When we decrease illumination, $r_{M}^{*}$ decreases and the temperature where the peak is located decreases. Second, we want to point out that the maximum of the expression $\left(1-A^{\prime *}-B^{\prime *}\right)_{M}$ is close to 1 when considering the value of the ratio $S_{n}^{*} / S_{r}^{*}$ to be 100 , but it is closer to 1 the higher this ratio is.

If we now consider both terms of $R$ together in the case of a sample with a high excess of acceptors, in which case $\xi_{0}$ goes to -1 at low temperature, we obtain for $R$ a value of about -2 , which means that the gain is twice as large than the maximum gain obtainable in a photorefractive material without applied field considering Kukhtarev's model with only one type of carrier. At high grating spacing with $k^{2} \ll k_{0}^{2} / A^{\prime *}$, for the spacecharge field we obtain:

$$
E_{1}=2 i m k \frac{k_{B} T}{e}=2 i m E_{d},
$$

where $E_{d}$ is the diffusion field.

The enhancement of the gain due to the metastable state can be of a factor of 2 in ideal conditions. This enhancement is characteristic of the model we develop here. All the band transport models derived from Kukhtarev's model including both carriers ${ }^{17}$ or secondary traps ${ }^{2-4,29}$ predict a reduction of the photorefractive effect compared to the ideal one-defect-one-carrier model. In the real case the effect of temperature is more complicated, as both quantities $\xi_{0}$ and $\left(1-A^{\prime *}-B^{\prime *}\right)$ vary together with temperature. For example, the value

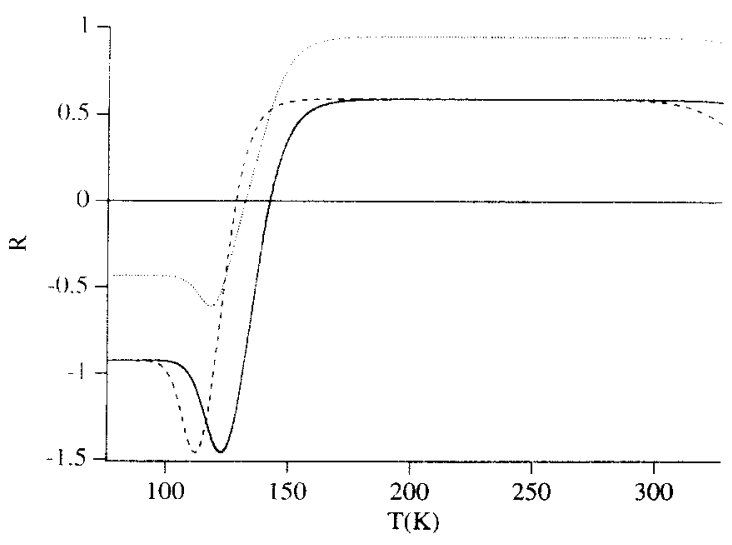

FIG. 3. Calculated coefficient $R$ as a function of temperature. Straight line: illumination $I=60 \mathrm{~mW} \mathrm{~cm}^{-2},[E L 2]=1.3 \times 10^{16}$ $\mathrm{cm}^{-3}$, and $\left[E L 2^{+}\right]=6 \times 10^{15} \mathrm{~cm}^{-3}$. Dashed line: illumination $I=4.3 \quad \mathrm{~mW} \mathrm{~cm}^{-2}, \quad[E L 2]=1.3 \times 10^{16} \quad \mathrm{~cm}^{-3}, \quad$ and $\left[E L 2^{+}\right]=6 \times 10^{15} \mathrm{~cm}^{-3}$. Dotted line: illumination $I=60$ $\mathrm{mW} \mathrm{cm}{ }^{-2},[E L 2]=1.3 \times 10^{16} \mathrm{~cm}^{-3}$, and $\left[E L 2^{+}\right]=1 \times 10^{15}$ $\mathrm{cm}^{-3}$. 
of the peak of $\left(1-A^{\prime *}-B^{\prime *}\right)$ is obtained for a value $A^{\prime *}=\sqrt{S_{r}^{*} /\left(S_{r}^{*}+S_{n}^{*}\right)}$ which is about $\frac{1}{10}$, this means that at this temperature the change of $\xi_{0}$ is not at a maximum. Nevertheless all the features predicted are preserved, particularly the possibility of having $|R|$ greater than 1 , even if the maximum value of 2 is hardly obtainable (Fig. 3).

Decreasing the temperature, we observe drastic changes of the PRE in GaAs due to the metastable state. Those changes are strongly dependent on the compensation ratio of the sample, going from a simple cancellation of the PRE to a change of the sign of the effect with an enhancement of the PRE which can reach a factor 2.

\section{Quenching of absorption and absorption grating}

The change of absorption is the main effect of the photoquenching of $E L 2$. This absorption change is due to redistribution of the population of the EL2 trap. Changes in the spatially modulated populations are also predicted by the model (with the result of the creation of the space-charge field). They will create a spatially modulated variation of the absorption. The aim of this section is to study these absorption gratings, as well as the linear absorption quenching.

According to our model, the nonmodulated part of absorption is

$\alpha_{0}=\left(S_{n}+S_{n}^{*}\right)\left(N_{T}-N_{0}-N_{0}^{*}\right)+S_{p} N_{0}+S_{r}^{*} N_{0}^{*}$,

whereas the spatially modulated part is

$$
\alpha_{1}=-\left(S_{n}+S_{n}^{*}\right)\left(N_{1}+N_{1}^{*}\right)+S_{p} N_{1}+S_{r}^{*} N_{1}^{*} \text {. }
$$

At $1.047 \mu \mathrm{m}$, the first term of linear absorption is predominant, whereas the third term can be neglected as $S_{r}^{*}$ is very low. When the temperature decreases from room temperature, the quenching of absorption occurs in two steps: at first, $N_{T}-N_{0}-N_{0}^{*}$ decreases, the main term of absorption disappears, and $\alpha_{0}$ equals $S_{p} N_{0}$; then, below $70 \mathrm{~K}, N_{0}$ decreases and absorption disappears totally.

From the material equations (the Appendix) we deduce $N_{1}$ and $N_{1}^{*}$ :

$N_{1}+N_{1}^{*}=A^{\prime *} N_{1}+m\left(1-A^{\prime *}-B^{\prime *}\right)\left(N_{T}-N_{0}-N_{0}^{*}\right)$,

$N_{1}=i \frac{k \varepsilon}{e} E_{1}$,

which for $\alpha_{1}$ gives

$$
\begin{aligned}
\alpha_{1}= & {\left[S_{p}-\left(S_{n}+S_{n}^{*}\right) A^{\prime *}+S_{r}^{*}\left(A^{\prime *}-1\right)\right]\left[i \frac{k \varepsilon}{e} E_{1}\right) } \\
& +m\left(1-A^{\prime *}-B^{\prime *}\right)\left(S_{r}^{*}-S_{n}-S_{n}^{*}\right)\left(N_{T}-N_{0}-N_{0}^{*}\right),
\end{aligned}
$$

with $E_{1}$ previously defined (3.10)

The first term is the classical absorption grating term. At room temperature it becomes $\left(S_{p}-S_{n}-S_{n}^{*}\right)\left[i(k \varepsilon / e) E_{1}\right]$. This term is generally very

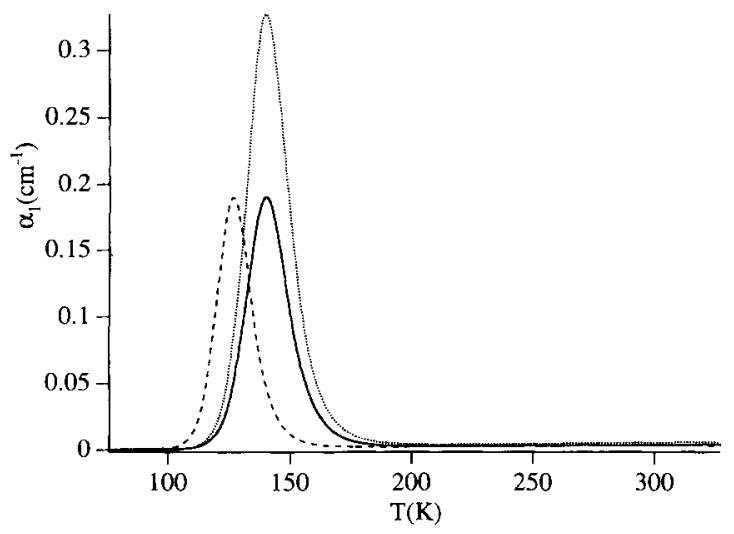

FIG. 4. Calculated amplitude of the absorption grating $\alpha_{1}$ as a function of temperature. Straight line: illumination $I=60$ $\mathrm{mW} \mathrm{cm}{ }^{-2},[E L 2]=1.3 \times 10^{16} \mathrm{~cm}^{-3}$, and $\left[E L 2^{+}\right]=6 \times 10^{15}$ $\mathrm{cm}^{-3}$. Dashed line: illumination $I=4.3 \mathrm{~mW} \mathrm{~cm}^{-2}$, $[E L 2]=1.3 \times 10^{16} \mathrm{~cm}^{-3}$, and $\left[E L 2^{+}\right]=6 \times 10^{15} \mathrm{~cm}^{-3}$. Dotted line: illumination $I=60 \mathrm{~mW} \mathrm{~cm}{ }^{-2},[E L 2]=1.3 \times 10^{16} \mathrm{~cm}^{-3}$, and $\left[E L 2^{+}\right]=1 \times 10^{15} \mathrm{~cm}^{-3}$.

small and hardly observable in undoped GaAs. ${ }^{30}$ With decreasing temperature the increase of $E_{1}$ and the variation of the cross-section factor are small, which means that this term induces an absorption grating of very low amplitude whatever the temperature. The second term is more interesting, as it vanishes at room temperature $\left(1-A^{\prime *}-B^{\prime *}=0\right)$ and is directly proportional to the $E L 2^{0}$ concentration. As previously seen, $\left(1-A^{\prime *}-B^{\prime *}\right)$ goes to a maximum at low temperature and $N_{T}-N_{0}-N_{0}^{*}$ constantly decreases. We thus observe a competition between these two factors, resulting in an absorption grating peak (Fig. 4) which can be very impor$\operatorname{tant}\left(\alpha_{1 M} \approx 0.2 \mathrm{~cm}^{-1}\right)$.

As a conclusion we observe theoretically an absorption grating peak, which leads to high diffraction efficiency or a beam-coupling energy transfer. The peak is very narrow in temperature (Fig. 4), and its position depends on illumination. Its height depends only on the $E L 2^{0}$ concentration, which varies only a little from one sample to another (by a factor less than 2). This absorption grating should be observed in the same way in different samples with different occupation ratios of $E L 2$, contrary to the PRE which depends strongly on the ratio of $E L 2^{+}$and $E L 2^{\circ}$. Moreover, as the absorption grating does not depend on $E L 2^{+}$concentration, it should be observed in $n$ type crystals where all $E L 2$ is in the neutral state and where no PRE is observed.

\section{EXPERIMENTAL ASPECT}

\section{A. Two-beam coupling}

As seen in Sec. III, the interference of two coherent beams on a photorefractive crystal creates a space-charge redistribution that induces a spatially modulated spacecharge field. This space-charge field is transformed through the Pockels effect in an index grating. ${ }^{16}$ The amplitude of this index grating is 


$$
\Delta n=\frac{n_{0}^{3} r_{\mathrm{eff}}\left|E_{1}\right|}{2},
$$

with $\left|E_{1}\right|$ the amplitude of the spatially modulated space-charge field (3.10). $r_{\text {eff }}$ is the effective electrooptic coefficient which depends on both the orientation of the sample and the polarization of the beams, ${ }^{30,31}$ and $n_{0}$ is the linear refractive index of the material (Table I). In the classical "beam-coupling" orientation, i.e., the propagation along [ $\overline{1} 10]$, grating vector along [001], and polarization along [110], we have $r_{\text {eff }}=r_{41}$ (Table I).

In order to measure this index variation we choose the two-beam-coupling technique for our measurements. The two beams that create the grating diffract on it, leading to an energy transfer from one beam to the other. ${ }^{16}$ In the presence of the pump beam $I_{p}$, the transmission of the probe beam, $I_{s}$, for a crystal of thickness $d$, is $I_{s}=I_{s 0} e^{-\left(\alpha_{0}+\Delta \alpha+\Gamma\right) d}$ (for small modulation of the grating, i.e., $\left.I_{s} \ll I_{p}\right)$, with $\Gamma=\left(2 \pi n_{0}^{3} r_{\text {eff }} / \lambda \cos \theta\right) \operatorname{Im}\left(E_{1} / m\right)$ the photorefractive gain $(\theta$ is the half angle of the interfering beams within the sample), $\Delta \alpha=\operatorname{Re}\left(\alpha_{1} / m\right)$ the absorption grating gain, and $\alpha_{0}$ the linear absorption. The photorefractive gain is optimum when the space charge field is purely imaginary, as it is the case in our experiments (3.10). When the pump beam is cut, no grating is present and we have $I_{s}=I_{s 0} e^{-\alpha_{0} d}$. We can then define the effective gain $\gamma_{0}$ that characterizes the energy transfer as the ratio of the probe beam intensity in the presence of the pump beam to the probe beam intensity without the pump beam:

$$
\gamma_{0}=e^{-(\Delta \alpha+\Gamma) d} \text {. }
$$

In order to separate the photorefractive gain from the absorption grating gain, we used the properties of symmetry of the PRE. For example, in the "beam-coupling" configuration, turning the crystal by $180^{\circ}$ around the [110] axis changes the sign of $\Gamma$, whereas the absorption grating gain $\Delta \alpha$ retains the same sign. Thus two measurements in the two orientations allow us to extract the photorefractive gain $\Gamma$ on the one hand and the absorption grating $\Delta \alpha$ on the other hand. In all our experimental measurements we make this treatment, and present $\Gamma$ and $\Delta \alpha$ directly.

\section{B. Setup and sample}

The experiments were performed with a diode-pumped Nd:YLF laser (emitting at $1.047 \mu \mathrm{m}$ ). The laser was split into two $s$-polarized beams that intersected inside the sample, producing a sinusoidal figure of interference with a grating spacing $\Lambda=3 \mu \mathrm{m}$ (Fig. 5). The pump beam was chopped at a frequency of about $7 \mathrm{~Hz}$. The probe beam was attenuated in order to have a pump-to-probe beam ratio of about 100. After the sample the probe beam was detected with a silicon photodiode placed after a spatial filtering that eliminated eventual scattering. The typical total illumination used in the experiment was 60 $\mathrm{mW} \mathrm{cm}{ }^{-2}$ inside the crystal.

The sample was placed in a liquid-nitrogen cryostat and cooled under illumination. In order to avoid the vibration induced by the boiling nitrogen, the measurement was performed during warming up of the cryostat, after all the nitrogen was evaporated. The temperature range of the experiment was 90-300 K. The warming of the sample occurred with a maximum rate of about 2 $\mathrm{K} \mathrm{mn}^{-1}$. Around room temperature a resistor was used to heat the sample. The temperature was measured with a nickel-chromium thermocouple placed on the sample carrier. We were not able to control the temperature and stabilize it at a fixed position, but the temperature variation was slow enough to allow the measurement of temperature at each instant and to ensure that the sample was in thermal equilibrium.

The beam-coupling energy transfer was very low, typically some percent, which means that when the pump beam was chopped the intensity variation of the probe beam was small. The period of the chopper was chosen to be much greater than the response time of the photorefractive effect in GaAs. The signal of the detector was electronically separated into two parts. One part

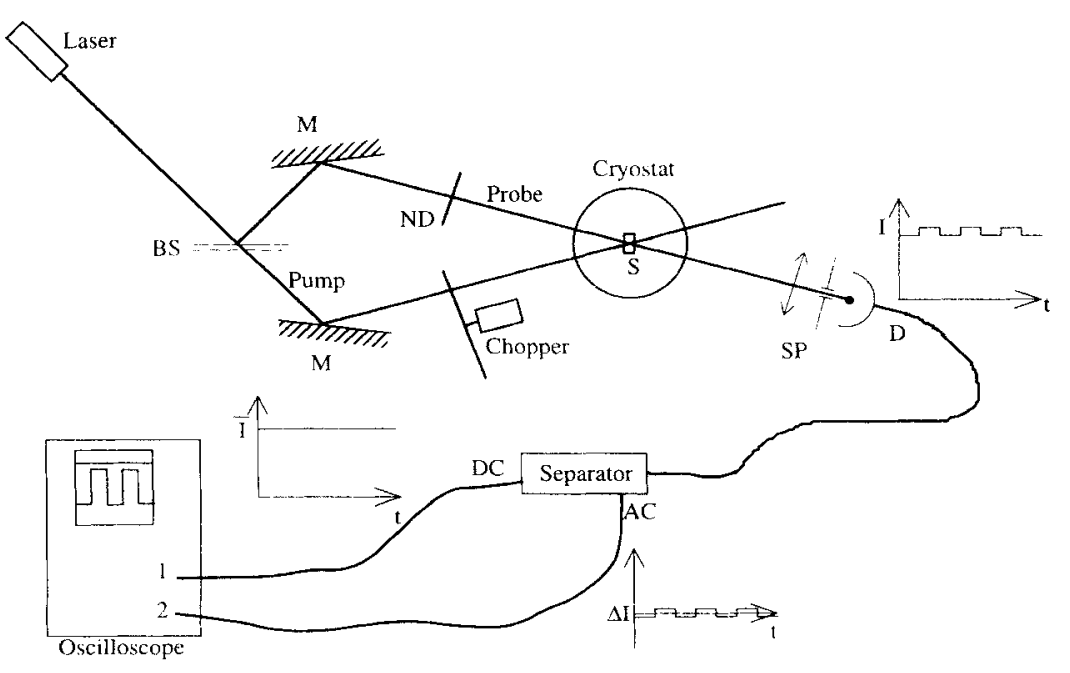

FIG. 5. Experimental setup. BS: beam splitter. ND: neutral density. SP: spatial filtering. $D$ : detector. $S$ : sample. $M$ : Mirror. 
passed through a long-time constant integrator $(\tau \approx 100$ s), the other part was subtracted from the integrated part in order to extract the component modulated with the chopper frequency. The two resulting signals, the mean value of the signal $\bar{I}$ and the modulated part $\Delta I$, were sent on the two inputs of an oscilloscope. A computer controlled the entire experiment, and at each instant (every two or three seconds) the temperature of the sample as well as $\bar{I}$ and $\Delta I$ were recorded. We thus obtained $\gamma_{0}=(2 \bar{I}-\Delta I) /(2 \bar{I}+\Delta I)$ as a function of temperature. For the treatment we made an average of the obtained values on small temperature intervals $(\approx 0.5 \mathrm{~K})$ in order to compare curves for different orientations of the sample and then extract the photorefractive part and the absorption part of the energy transfer.

We used two undoped GaAs samples with close characteristics, i.e., high excess of shallow acceptors and $\left[E L 2^{0}\right] \approx\left[E L 2^{+}\right]$, and close photorefractive performance. The only difference between the two samples was the orientation of the crystallographic axes. The first sample $D 2$ was cut with the classical photorefractive configuration, the beams propagated in the $[\overline{1} 10]$ direction, with a grating vector along [001] and polarization along [110], which for this configuration gives $r_{\text {eff }}=r_{41}$. The thickness of this sample was $d=1.72 \mathrm{~mm}$. Absorption, electron paramagnetic resonance (EPR), and roomtemperature photorefractive measurements performed with this sample 25 gave $[E L 2]=1.3 \times 10^{16} \mathrm{~cm}^{-3}$ and $\left[E L 2^{+}\right]=6 \times 10^{15} \mathrm{~cm}^{-3}$. For the second sample $C 4$ the beam propagated along the [111] direction, with grating vector along $[11 \overline{2}]$ and polarization along $[\overline{1} 10]$, which for this configuration gives $r_{\text {eff }}=\sqrt{\frac{2}{3}} r_{41} .25,30$ Its thickness was $d=1.83 \mathrm{~mm}$.

\section{Experimental results}

The behavior of the photorefractive gain $\Gamma$ corresponds exactly to the theory (Fig. 6). First, between 300

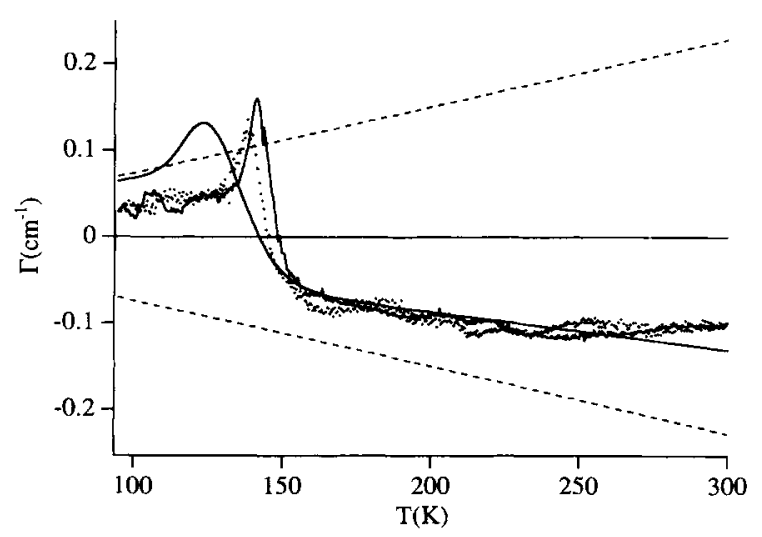

FIG. 6. Experimental gain $\Gamma$ as a function of temperature for sample $D 2$ (dots) and sample $C 4$ (line) normalized to the same value of effective electro-optic coefficient as $D 2$. The straight line represents the calculated gain (see text for calculation parameters). The dashed lines represents the theoretical gain for a one-carrier model corresponding to a space-charge field of amplitude $\pm E_{d}$.

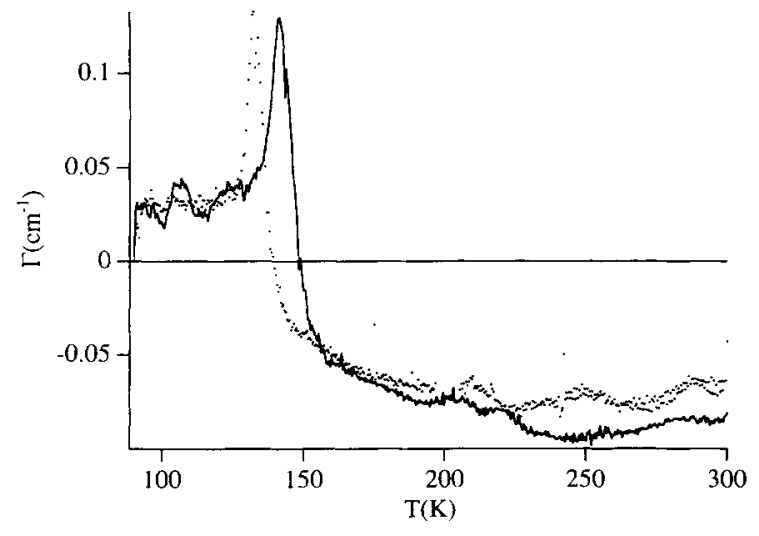

FIG. 7. Experimental gain $\Gamma$ in sample $C 4$ for two different intensities. $I=60 \mathrm{~mW} \mathrm{~cm}^{-2}$ (line) and $I=4.3 \mathrm{~mW} \mathrm{~cm}^{-2}$ (dots), showing the displacement of the peak toward lower temperature, with decreasing intensity.

and $150 \mathrm{~K}$ a small decrease corresponding to the decrease of the diffusion field with temperature is observed. At $150 \mathrm{~K}$ a strong decrease of the gain occurs, followed by a change of the sign. This reverse of the sign is followed by a peak of gain centered around $140 \mathrm{~K}$, the maximum value being around $0.12-0.15 \mathrm{~cm}^{-1}$. After this peak the gain decreases linearly with temperature. The same feature is observed in the two samples studied, as shown in Fig. 6, where the gain of the two samples is presented, the value for $C 4$ being corrected for the $\sqrt{\frac{2}{3}}$ ratio due to the configuration. This proves that the effect observed is purely a space-charge field effect. At this point we can remark that the result obtained here is strongly different from the results previously observed in the literature, ${ }^{7}$ we will return to this difference below. When decreasing the illumination on the sample (Fig. 7), we observe a displacement of the peak of the gain of about $10 \mathrm{~K}$ corresponding to what was predicted theoretically (Fig. 3).

The other effect we observe concerns the absorption part $\Delta \alpha$ of the beam coupling (Fig. 8). The absorption

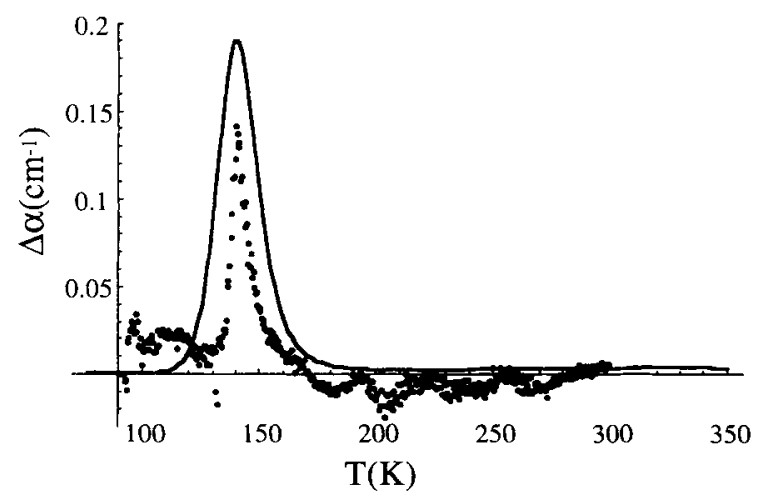

FIG. 8. Experimental absorption energy transfer $\Delta \alpha$ as a function of temperature (dots). The straight line represents the calculated absorption grating strength (same parameters as for Fig. 6). 
grating is negligible at room temperature, as expected in that type of material and as previously observed. ${ }^{30}$ At low temperature we see the appearance of a strong peak of the absorption part of the beam coupling centered at $140 \mathrm{~K}$. The absorption grating vanishes at lower temperature. This feature corresponds exactly to the theory (Fig. 8).

\section{Discussion}

All the parameters concerning the $E L 2$ defect (Table I) as well as the concentrations in our sample are known. We can thus calculate the variation of the gain with the theoretical expression (3.10) previously established, and compare it with the experimental results. The accordance between the experimental data and the theoretical model is good (Fig. 6). The principal point, which is the enhancement of the gain which corresponds to the space-charge field higher than the diffusion field, is well explained (Fig. 6). The same accordance between theory and experiment is observed for the absorption grating (Fig. 8).

Our theoretical model also explains the previous results obtained in $\mathrm{GaAs}^{7}$ even if these experimental results are very different from ours. The first point is that the sample used by Nolte, Olson, and Glass corresponds to a sample with a small excess of acceptors corresponding to a compensation ratio $\left[E L 2^{+}\right] /\left[E L 2^{0}\right] \approx 0.1$. This explains that the enhancement of the gain is not observed in this sample, as previously discussed. The second point is the absorption grating, which depends on the $E L 2^{0}$ concentration which is constant in LEC-grown samples around $1-2 \times 10^{16} \mathrm{~cm}^{-3}$. The fact that this absorption grating is not observed in Ref. 7 is due mainly to the fact that the experiment was a four-wave mixing experiment which was performed with a readout beam at $1.32 \mu \mathrm{m}$, a wavelength where the value of the different cross sections are strongly different from that at $1.047 \mu \mathrm{m}$. Indeed, in the expression for $\alpha_{1}[(3.14)]$, the reading wavelength cross sections are used. For these cross sections we have $S_{n}=1.5 \times 10^{-17} \mathrm{~cm}^{2}$ and $S_{p}=5 \times 10^{-17} \mathrm{~cm}^{2},^{18}$ and we can estimate $S_{n}^{*}=S_{n}^{*}(1.06 \mu \mathrm{m}) / 100 \quad$ (Ref. 32) and $S_{r}^{*}=0.6 S_{n}^{*} \cdot{ }^{22,26}$ For the space-charge field the value is

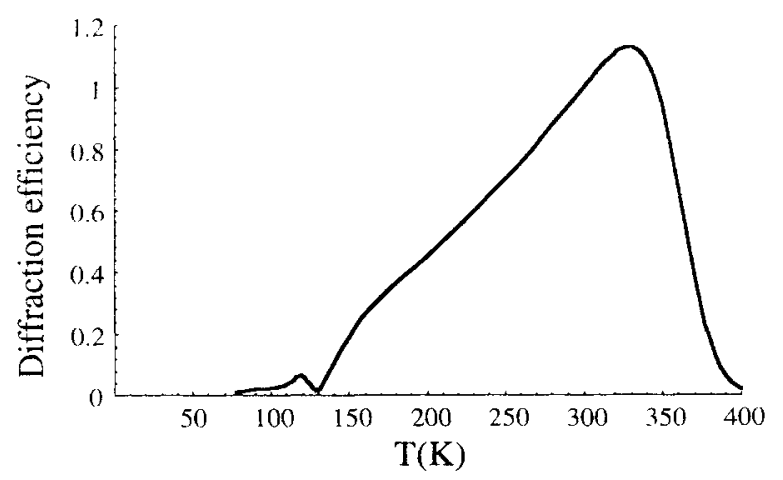

FIG. 9. Calculated diffraction efficiency for the experiment of Ref. 7. calculated with the writing beam wavelength (i.e., 1.06 $\mu \mathrm{m}$ ); only the value of the refractive index, the electrooptic coefficient, and the wavelength are changed: $n=3.42$ (Ref. 23) and $r_{41}=1.50 \mathrm{pm} \mathrm{V}^{-1},{ }^{24}$ in $\Delta n$ expression. We can then calculate the theoretical diffraction efficiency with the formula: ${ }^{6}$

$$
\eta=\sin ^{2}\left(\frac{\pi \Delta n d}{\lambda \cos \theta}\right)+\operatorname{sh}^{2}\left(\frac{\alpha_{1} d}{4 \cos \theta}\right) .
$$

The calculated gain corresponds to what was observed previously in Ref. 7 (Fig. 9). The small residual bump, which is not seen in that experiment, perhaps corresponds to values of diffraction efficiencies below the threshold of the detection of the experiment. Nevertheless the presence of this bump does not obliterate the general agreement between the two curves.

\section{CONCLUSION}

Photorefractive two-beam-coupling experiments performed at low temperature in undoped GaAs reveal peculiar characteristics of this material, such as a reversal of the sign of the photorefractive gain, strong enhancement of the value of this photorefractive gain, and a peak of the absorption grating at low temperature. These results are explained theoretically by the introduction, in the photorefractive model, of the well-known metastable state of the EL2 defect that appears at low temperature. All experimental characteristics of the photorefractive effect are perfectly described by this model, in particular the fact that space-charge fields higher than the diffusion field are observed. These first results show the interest of DLPS studies in the case of GaAs. The study of different samples with variable wavelength will provide information about the optical properties of the metastable state of $E L 2$ and consequently about the microscopic structure of this defect. Even $n$-type samples, where no spacecharge field can be created, could be studied by this technique due to the presence of the absorption grating. This study also shows that the photorefractive gain obtained by taking into account only one defect and one type of charge carrier can be overcome in particular cases such as the one presented here. Further development of the model could be performed, especially concerning the time dependence of the effect, an aspect not discussed here.

\section{ACKNOWLEDGMENTS}

Ph.D. wants to thank the Universität Osnabrück for its financial support and Dr. R. Rupp for his welcome at the Fachbereich Physik. Financial support of the VW foundation (Project No. ODU63) and of the Deutsche Forschungsgemeinschaft (SFB225, Project No. A6) are gratefully acknowledged.

\section{APPENDIX}

In this appendix a calculation of the space-charge field $E_{1}$ is given. The first Fourier components of the equation set $(3.1 \mathrm{a})-(3.1 \mathrm{~g})$ are 


$$
\begin{aligned}
0= & \left(\beta_{n}+S_{n} I_{0}\right)\left(-N_{1}-N_{1}^{*}\right)+S_{n} I_{0} m\left(N_{T}-N_{0}-N_{0}^{*}\right) \\
& -\gamma_{n} n_{0} N_{1}-\gamma_{n} n_{1} N_{0}-\left(\beta_{p}+S_{p} I_{0}\right) N_{1}-S_{p} I_{0} m N_{0} \\
& +\gamma_{p} p_{0}\left(-N_{1}-N_{1}^{*}\right)+\gamma_{p} p_{1}\left(N_{T}-N_{0}-N_{0}^{*}\right), \quad \text { (A1a) } \\
0= & S_{n}^{*} I_{0}\left(-N_{1}-N_{1}^{*}\right)+S_{n}^{*} I_{0} m\left(N_{T}-N_{0}-N_{0}^{*}\right) \\
& -\left(r^{*}+S_{r}^{*} I_{0}\right) N_{1}^{*}-S_{r}^{*} I_{0} m N_{0}^{*}, \\
j_{n 1}= & e n_{0} \mu_{n} E_{1}+e n_{1} \mu_{n} E_{0}+i k \mu_{n} k_{B} T n_{1}, \\
j_{p 1}= & e p_{0} \mu_{p} E_{1}+e p_{1} \mu_{p} E_{0}-i k \mu_{p} k_{B} T p_{1}, \\
0= & \frac{i k}{e} j_{n 1}+\left(\beta_{n}+S_{n} I_{0}\right)\left(-N_{1}-N_{1}^{*}\right) \\
& +S_{n} I_{0} m\left(N_{T}-N_{0}-N_{0}^{*}\right)-\gamma_{n} n_{0} N_{1}-\gamma_{n} n_{1} N_{0},
\end{aligned}
$$

From Eqs. (A1a), (A1e), and (A1f) it directly follows that

$$
j_{n 1}+j_{p 1}=0 \text {. }
$$

In the following we assume an externally applied field to be absent $\left(E_{0}=0\right)$. Inserting (A1d) into (A1f) [(A1c) into (A1e)] with the above-mentioned abbreviations $\alpha_{p}$, $A_{p}$, and $\kappa_{p}\left(\alpha_{n}, A_{n}\right.$, and $\left.\kappa_{n}\right)$, we obtain the expression for $p_{1}\left(n_{1}\right)$ as a function of $E_{1}, N_{1}$, and $N_{1}^{*}$ :

$p_{1}=\frac{-i k p_{0} \mu_{p} E_{1}+A_{p} S_{p} N_{1} I_{0}+I_{0} m \alpha_{p}+\gamma_{p} p_{0}\left(N_{1}+N_{1}^{*}\right)}{\frac{k_{B} T}{e} \mu_{p}\left(k^{2}+\kappa_{p}^{2}\right)}$,

$n_{1}=\frac{i k n_{0} \mu_{n} E_{1}-A_{n} S_{n}\left(N_{1}+N_{1}^{*}\right) I_{0}+I_{0} m \alpha_{n}-\gamma_{n} n_{0} N_{1}}{\frac{k_{B} T}{e} \mu_{n}\left(k^{2}+\kappa_{n}^{2}\right)}$

We now want to express every component $X_{1}$ as a function of $E_{1}$. From (A1g) in the limit $n_{1}, p_{1} \ll N_{1}$, we obtain

$$
N_{1}=\frac{i k \varepsilon}{e} E_{1} .
$$

Finally, (A 1b) related $N_{1}^{*}$ and $N_{1}$ as

$$
N_{1}^{*}=\frac{-S_{n}^{*} I_{0} N_{1}+S_{n}^{*} I_{0} m\left(N_{T}-N_{0}-N_{0}^{*}\right)-S_{r}^{*} I_{0} m N_{0}^{*}}{r^{*}+S_{r}^{*} I_{0}+S_{n}^{*} I_{0}}
$$

which may be rewritten as

$$
N_{1}+N_{1}^{*}=A^{\prime *} N_{1}+m\left(1-A^{\prime *}-B^{\prime *}\right)\left(N_{T}-N_{0}-N_{0}^{*}\right) \text {, }
$$

which proves Eq. (3.15). So Eqs. (A5) and (A7) enable us also to express $p_{1}$ and $n_{1}$ as functions of $E_{1}$. Subtracting Eq. (A1f) from (A1e) under the consideration of (A2), an equation involving the quantities $n_{1}, p_{1}, N_{1}$ and $N_{1}^{*}$, is obtained

$$
\begin{aligned}
0= & -A_{n} S_{n}\left(N_{1}+N_{1}^{*}\right) I_{0}+I_{0} m \alpha_{n}-\gamma_{n} n_{0} N_{1} \\
& -n_{1} \kappa_{n}^{2} \frac{k_{B} T}{e} \mu_{n}-A_{p} S_{p} N_{1} I_{0}-I_{0} m \alpha_{p} \\
& -\gamma_{p} p_{0}\left(N_{1}+N_{1}^{*}\right)+p_{1} \kappa_{p}^{2} \frac{k_{B} T}{e} \mu_{p} .
\end{aligned}
$$

Now inserting for $n_{1}, p_{1}, N_{1}$ and $N_{1}+N_{1}^{*}$ the expressions (A3), (A4), (A5), and (A7), respectively, we obtain an equation depending only on $E_{1}$. Taking account of $S_{n}=\alpha_{n} /\left(N_{T}-N_{0}\right) A^{\prime *}$ and $S_{p}=\alpha_{p} / N_{0}$ and the zeroth Fourier components $n_{0}$ and $p_{0}$ given in Eqs. (3.4) and (3.5), respectively, after some algebra the desired spacecharge field $E_{1}$ can be written as

$$
E_{1}=\frac{-i m \frac{k_{B} T}{e} k}{1+A^{\prime *} \frac{k^{2}}{k_{0}^{2}}}\left[\xi(k)-\left(1-A^{\prime *}-B^{\prime *}\right)\right],
$$

with the already mentioned electron-hole competition coefficient $\xi$.
${ }^{1}$ P. Günter and J. P. Huignard, Photorefractive Materials and Their Applications I. Fundamental Phenomena, Topics in Applied Physics Vol. 61 (Springer-Verlag, Berlin, 1988); Photorefractive Materials and Their Applications II. Survey of Applications, Topics in Applied Physics Vol. 62 (SpringerVerlag, Berlin, 1989).

${ }^{2}$ Ph. Delaye, H. J. Von Bardeleben, and G. Roosen, Appl. Phys. A 59, 357 (1994).

${ }^{3}$ P. Delaye, P. U. Halter, and G. Roosen, J. Opt. Soc. Am. B 7, 2268 (1990).

${ }^{4}$ R. S. Rana, D. D. Nolte, R. Steldt, and E. M. Monberg, J. Opt. Soc. Am. B 9, 1614 (1992).
5J. C. Launay, V. Mazoyer, M. Tapiero, J. P. Zielinger, Z. Guellil, Ph. Delaye, and G. Roosen, Appl. Phys. A 55, 33 (1992).

${ }^{6}$ D. D. Nolte, D. H. Olson, and A. M. Glass, Appl. Phys. Lett. 56, 163 (1990).

${ }^{7}$ D. D. Nolte, D. H. Olson, and A. M. Glass, Phys. Rev. B 40, 10650 (1989).

${ }^{8}$ A. L. Khromov and M. P. Petrov, Opt. Commun. 88, 315 (1992).

${ }^{9}$ G. M. Martin, J. P. Farges, G. Jacob, J. P. Hallais, and G. Poiblaud, J. Appl. Phys. 51, 2840 (1980).

${ }^{10}$ E. J. Johnson, J. A. Kafalas, and R. W. Davies, J. Appl. Phys. 54, 204 (1983). 
11 J. C. Bourgoin, H. J. von Bardeleben, and D. Stiévenard, J. Appl. Phys. 64, R65 (1988).

${ }^{12}$ M. O. Manasreh, D. W. Fischer, and W. C. Mitchel, Phys. Status Solidi B 154, 11 (1989).

${ }^{13}$ G. Vincent, D. Bois, and A. Chantre, J. Appl. Phys. 53, 3643 (1982).

${ }^{14}$ G. M. Martin, Appl. Phys. Lett. 39, 747 (1981).

${ }^{15}$ H. J. von Bardeleben, D. Stiévenard, D. Deresmes, A. Huber, and J. C. Bourgoin, Phys. Rev. B 34, 7192 (1986).

${ }^{16}$ N. V. Kukhtarev, V. B. Markov, S. G. Odulov, M. S. Soskin, and V. L. Vinetskii, Ferroelectrics 22, 949 (1979); 22, 961 (1979).

${ }^{17}$ F. P. Strohkendl, J. M. C. Jonathan, and R. W. Hellwarth, Opt. Lett. 11, 312 (1986).

${ }^{18}$ P. Silverberg, P. Omling, and L. Samuelson, Appl. Phys. Lett. 52, 1689 (1988).

${ }^{19}$ V. Y. Prinz and S. N. Rechkunov, Phys. Status Solidi B 118, 159 (1983).

20J. S. Blakemore, J. Appl. Phys. 53, R123 (1982).
${ }^{21}$ J. S. Blakemore, J. Phys. Chem. Solids 49, 627 (1988).

${ }^{22}$ M. O. Manasreh and D. W. Fisher, Phys. Rev. B 40, 11756 (1989).

${ }^{23}$ D. T. F. Marple, J. Appl. Phys. 35, 1241 (1964).

${ }^{24}$ C. A. Berseth, C. Wuethrich, and F. K. Reinhart, J. Appl. Phys. 71, 2821 (1992).

${ }^{25} \mathrm{Ph}$. Delaye, thèse de doctorat, Université Paris XI Orsay, 1993 (unpublished).

${ }^{26}$ J. C. Parker and R. Bray, Phys. Rev. B 37, 6368 (1988).

${ }^{27}$ D. W. Fisher and M. O. Manasreh, Appl. Phys. Lett. 54, 2018 (1989).

${ }^{28}$ G. Picoli, P. Gravey, C. Ozkul, and V. Vieux, J. Appl Phys. 66, 3798 (1989).

${ }^{29}$ P. Tayebati, J. Opt. Soc. Am. B 9, 415 (1992).

${ }^{30}$ B. Sugg, F. Kahmann, R. A. Rupp, Ph. Delaye, and G. Roosen, Opt. Commun. 102, 6 (1993).

${ }^{31}$ J. C. Fabre, J. M. C. Jonathan, and G. Roosen, Opt. Commun. 65, 257 (1988).

${ }^{32}$ D. W. Fisher, Appl. Phys. Lett. 50, 2751 (1987). 